\title{
Geologic mapping and basement-sediment contact delineation along Profile X, Igarra-Auchi area, Southern Nigeria using ground magnetic and electromagnetic methods
}

\author{
Chidiebere Charles Agoha ${ }^{1} \cdot$ Tochukwu Innocent Mgbeojedo $^{2}$ (D) Eze Martins Okoro ${ }^{3} \cdot$ Francis Begianpuye Akiang $^{1}$. \\ Chukwuebuka Nnamdi Onwubuariri ${ }^{4}$. Latifa Shaheen AI-Naimi ${ }^{5}$
}

Received: 10 April 2021 / Accepted: 2 June 2021 / Published online: 22 June 2021

(C) The Author(s) 2021

\begin{abstract}
Outcrop mapping as well as electromagnetic and ground magnetic surveys was carried out within Auchi and Igarra localities in order to attempt an interpretation of the geology of the areas and to delineate the boundary between basement and sedimentary terrains. Geologic mapping was done by collecting samples of outcrops at five different locations within the areas. Three lithofacies were identified within Auchi area and they are the basal shale unit, tabular cross-bedded sandstone unit and ferruginized sandstone unit. The pebbly shale is greyish black in colour; the cross-bedded sandstone unit is greyish white, coarse-grained at the base and finer at the top with pockets of clay, while the ferruginized sandstone is dark red. Rocks of the Precambrian basement complex underlie Igarra area. The area is underlain by metasediments that have been intruded by igneous rocks. Results show the presence of three major groups of igneous and metamorphic rocks within the area, and they are the migmatite-gneiss complex, metasediments and porphyritic granites. The electromagnetic and ground magnetic data acquired along Profile X located along Auchi-Igarra-Ibillo road were processed using Microsoft Excel Software and the resulting plots delineated areas with lower electrical conductivities and higher magnetic susceptibilities, as well as areas with higher electrical conductivities and lower magnetic susceptibilities. The areas with lower electrical conductivities and higher magnetic susceptibilities are interpreted to be underlain by basement rocks, while the areas with higher electrical conductivities and lower magnetic susceptibilities are underlain by sedimentary rocks. The plots also delineated the most likely basement-sedimentary boundary in the area.
\end{abstract}

Keywords Geologic mapping · Basement-sediment boundary · Electromagnetic method · Electrical conductivity · Magnetic susceptibility

Tochukwu Innocent Mgbeojedo toomgbeojedo@gmail.com

1 Department of Geology, Federal University of Technology, Owerri, Nigeria

2 Geotechnical Department, Arab Center for Engineering Studies, Doha, Qatar

3 Department of Geology, University of Nigeria, Nsukka, Nigeria

4 Department of Physics, Michael Okpara University of Agriculture, Umudike, Nigeria

5 Department of Chemistry and Earth Sciences, Qatar University, Doha, Qatar

\section{Introduction}

The Auchi/Igarra area of Edo State situated within the southern region of Nigeria is an area that is geologically significant. Its geologic significance stems from the fact that Auchi lies within the western part of Anambra Basin which is located in the Lower Benue Trough and as such is underlain by rocks of purely sedimentary origin, while Igarra area is an area underlain by rocks of the Precambrian basement, the area consists of metasediments intruded by Pan-African igneous rocks (Egbuniwe and Ocan 2009).

Several authors including Umeji and Nwajide (2007), Adekoya et al. (2011) have studied the sediments of Auchi area using methods like geologic mapping, textural and mineralogical characterization and diagenetic studies among others. The makeup, structural elements and nature of the

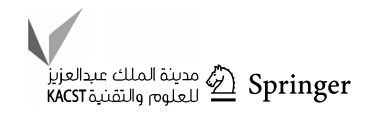


Precambrian basement rocks underlying Igarra area have also been studied by authors including Egbuniwe and Ocan (2009), Odeyemi and Rahaman (1992) and Oluseyi (2006). None of these authors was, however, able to delineate and establish the approximate contact or boundary between the basement terrain of Igarra area and the sedimentary environment of Auchi area.

The methods of investigation in this study involved field geologic mapping, electromagnetic survey using the very low-frequency (VLF) method and ground magnetic survey. Field geologic mapping is a method which entails studying rocks and geologic structures where they are best exposed and most easily interpreted. Electromagnetic method, on the other hand, is a geophysical survey technique that applies the principle of electromagnetic induction to measure the electrical conductivity of the subsurface (Kearey et al. 2002). The magnetic method is a geophysical survey technique which is used to investigate subsurface geology based on anomalies in the Earth's magnetic field originating from the magnetic properties of the underlying rocks (Kearey et al. 2002). The electromagnetic method and the magnetic method are the most important and commonly used technique for studying the electrical conductivity and magnetic susceptibility of underlying rocks. Field geologic mapping of outcrops in this area will validate the fact that Auchi area is underlain by rocks of purely sedimentary origin and Igarra area is underlain by rocks of the Precambrian basement. Delineating the boundary between these geologic terrains by studying the electrical and magnetic properties of the underlying materials will enable solid mineral miners and

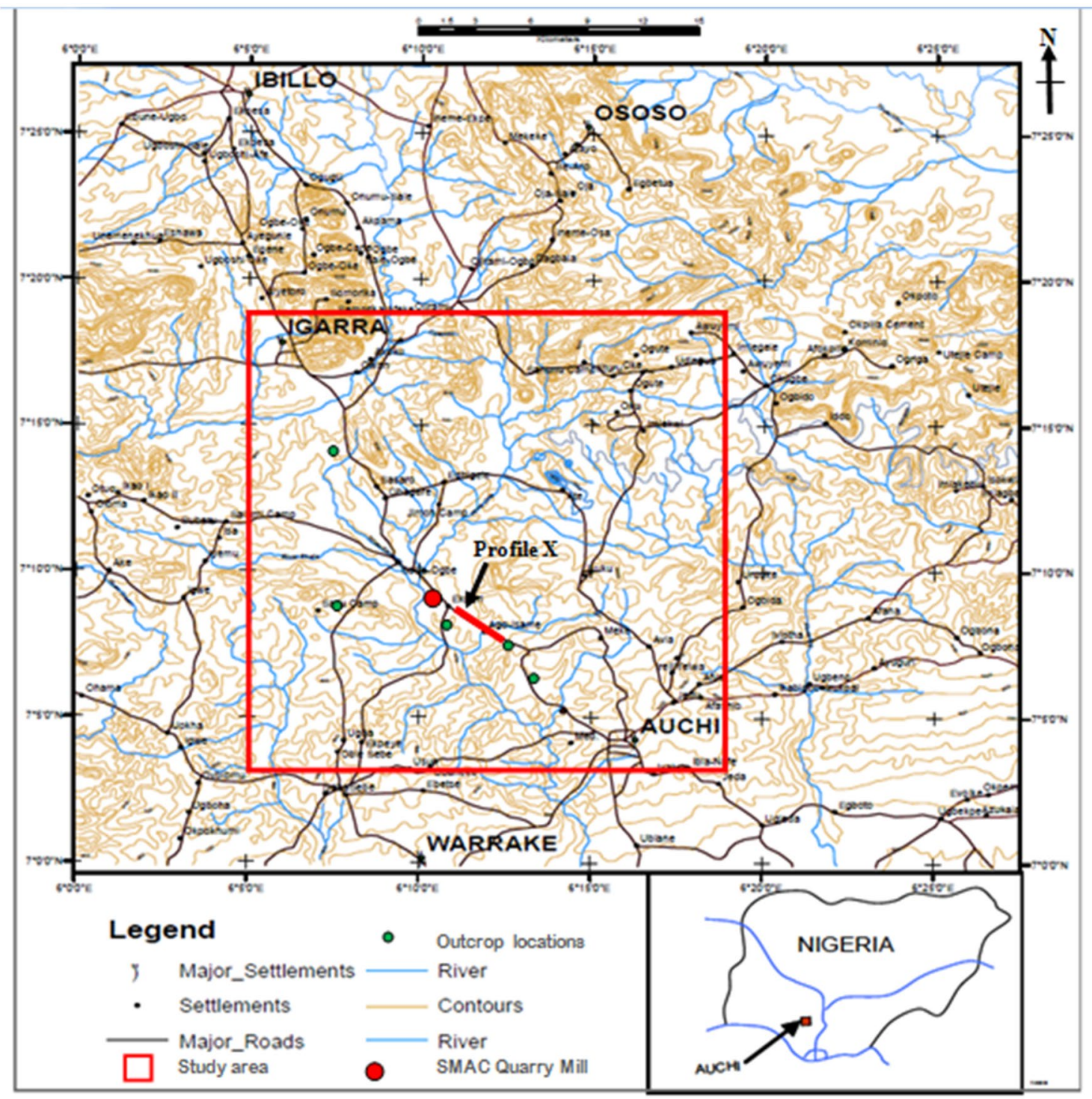

Fig.1 Location map showing the study area, including outcrop locations and profile X (Modified from Authors' extract of Auchi and environs (2012)) 
Fig.2 Sedimentary basins of Nigeria (after Obaje 2009)

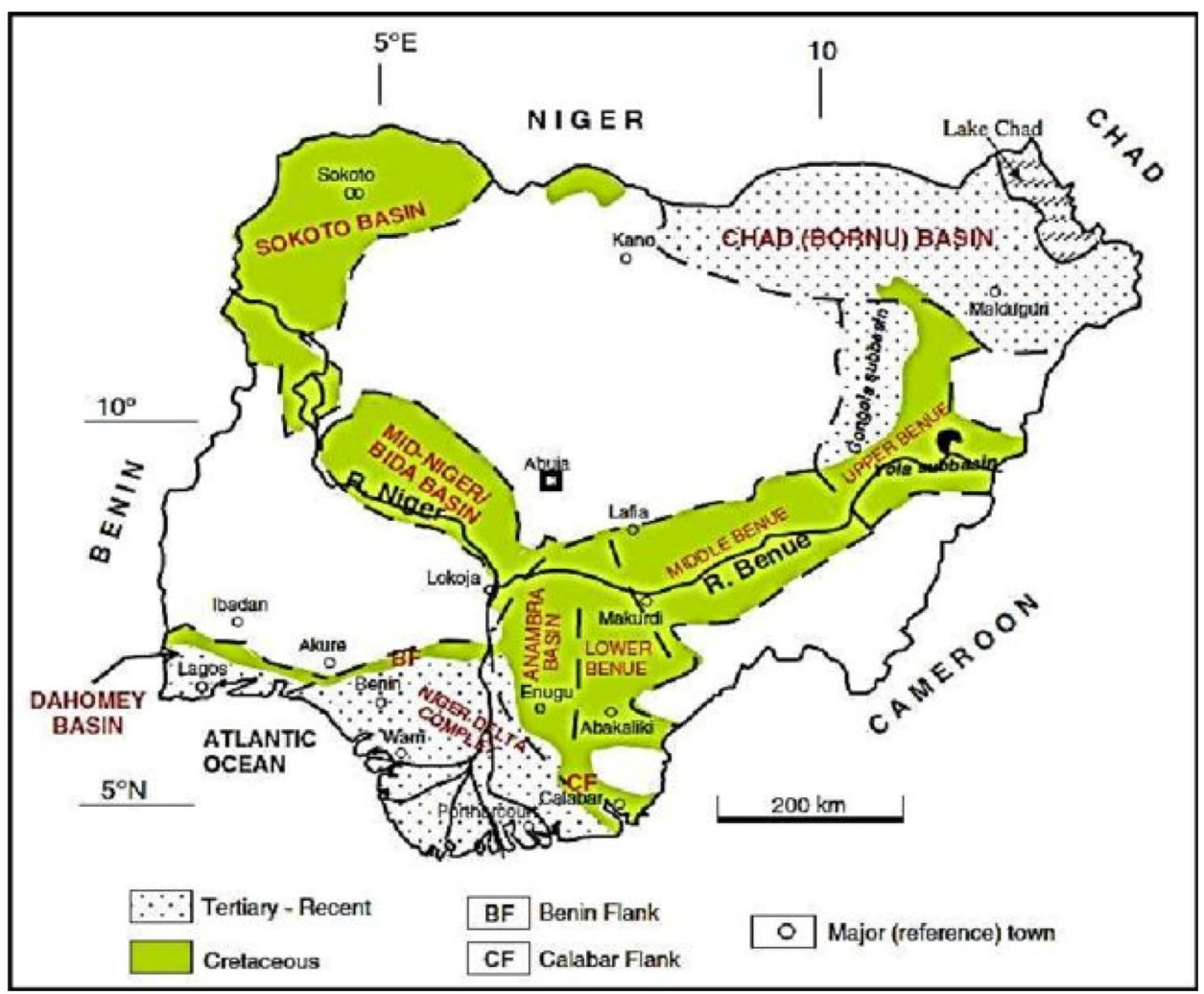

geologists involved in quarrying activities within Igarra area to have a good knowledge of the termination point of basement environment in the area. Knowledge of the boundary between these two terrains will also serve as a guide for drilling activities within these environments.

This work therefore presents a detailed account of the electrical conductivities and magnetic susceptibilities of the rocks underlying Auchi and Igarra areas leading to a better delineation of the boundary between the sedimentary and basement environments.

\section{Geology of the area}

Auchi and Igarra area, situated in Edo State Southern Nigeria, lies within latitude $7^{\circ} 04^{\prime} 00^{\prime \prime} \mathrm{N}$ and Longitude $6^{\circ} 16^{\prime} 00^{\prime \prime} \mathrm{E}$. Figure 1 shows the location map of the study area.

\section{Auchi}

Auchi area is located within the western portion of Anambra Basin. The shape of this basin is nearly triangular as shown in Fig. 3 and covers an area of about $3000 \mathrm{~km}^{2}$ with a sedimentary pile of approximate thickness of $9 \mathrm{~km}$ (Olubayo 2016; Iheanacho 2016). This basin is situated west of the lower Benue Trough as shown in Fig. 2 and according to Obaje (2009); it forms part of the Lower Benue Trough that contains sediments of Campanian-Maastrichtian to Eocene ages. Three lithofacies belonging to two different lithostratigraphic units are recognized in Auchi area, namely shale unit overlain by cross-bedded, burrowed sandstone which is then overlain by ferruginized sandstone (Adekoya et al. 2011). The shale unit is said to likely belong to Mamu Formation while the sandstone units are most likely the extension of Ajali Formation (Geologic map of Nigeria, GSN 1994). The depositional environment of the shale is believed to be shallow marine while that of the sandstone units is fluviodeltaic (Nwajide 1990). According to Ilegieuno et al. 2020, the sediments of Auchi area are fine- to coarse-grained and also poorly to moderately sorted.

Figure 2 shows the sedimentary basins of Nigeria after Obaje (2009), Fig. 3 shows the geologic map of Anambra Basin indicating the extension of Ajali sandstone within Auchi environs while Fig. 4 shows the stratigraphy and likely depositional environment of Anambra Basin modified from Tijani et al. (2010).

\section{Igarra}

Precambrian basement rocks, mainly metasediments intruded by igneous rocks of mostly pan-African age, underlie the entire Igarra area (Egbuniwe and Ocan 2009). According to Egbuniwe and Ocan (2009), this area is characterized by structural complexity due mainly to the different

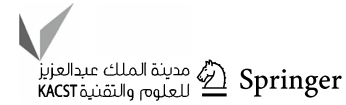




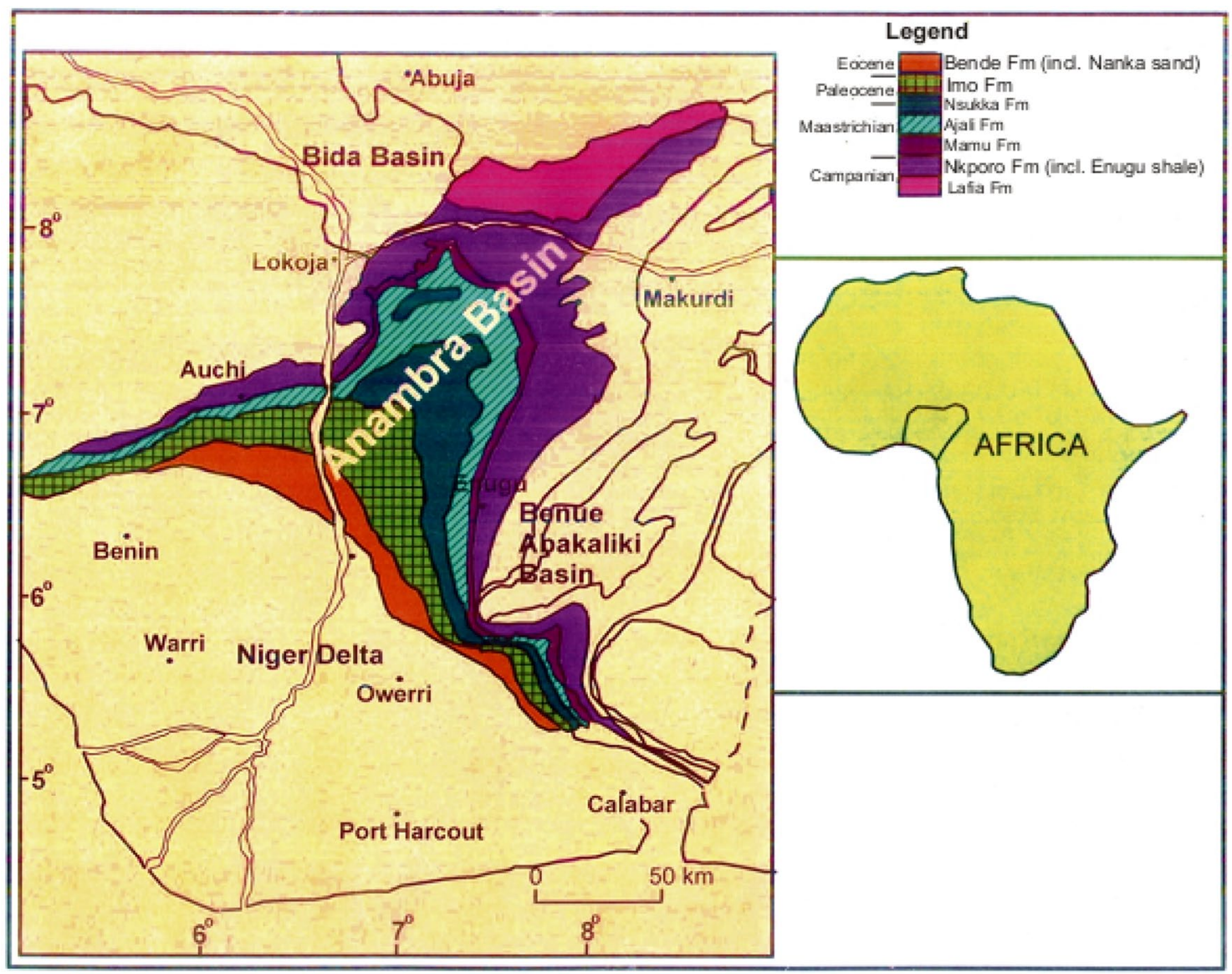

Fig.3 Geologic map of Anambra Basin showing the extension of Ajali sandstone within Auchi environs (Drawn from Geologic map of Nigeria, GSN 1994)

deformation components, including translation, rotation and strain, which produced structures during the Pan-African orogeny.

Underlying this area are metamorphic and igneous rocks classified into three major groups by Odeyemi and Rahaman (1992), namely the migmatite-gneiss complex, a sequence of upper Proterozoic metasediments and syn-to late-tectonic porphyritic granites shown in Fig. 5.

Granodiorites and syenites also exist in this area (Odeyemi and Rahaman 1992). From extensive study of the rocks of Igarra area, Odeyemi and Rahaman (1992) reported the existence of a discordance in structure between the older migmatite and both metasediment and older granite. This was due to the fact that most structural elements including folds and lineaments existing in this area have a common central axis with a dominant axis trending NW-SE in the metasediments and the older granites while the major structural element in the older migmatite trends E-W. This implies that there is no uniformity in the trend of structural elements in the rocks of this area. Marble, calc-gneiss, metaconglomerate, granites, pure quartz and kaolin occur in large quantities at different localities within Igarra area Oluseyi (2006). Figure 6 shows the geologic map of Igarra area after Anifowose et al. (2006).

\section{Materials and methods}

\section{Field geologic mapping}

The field geologic mapping covered a total of five localities within Auchi and Igarra areas from where samples were taken and examined. The materials used for this study include the Brunton Compass used to determine the direction of dip and strike of beds, sledge hammer to pick rock samples where they outcrop, hand lens to ascertain whether there are minerals within the rock, camera used for photographs and a small field knife for scratching the rock. At 
Fig.4 Stratigraphy and likely depositional environment of Anambra Basin ( Modified from Tijani et al. 2010)

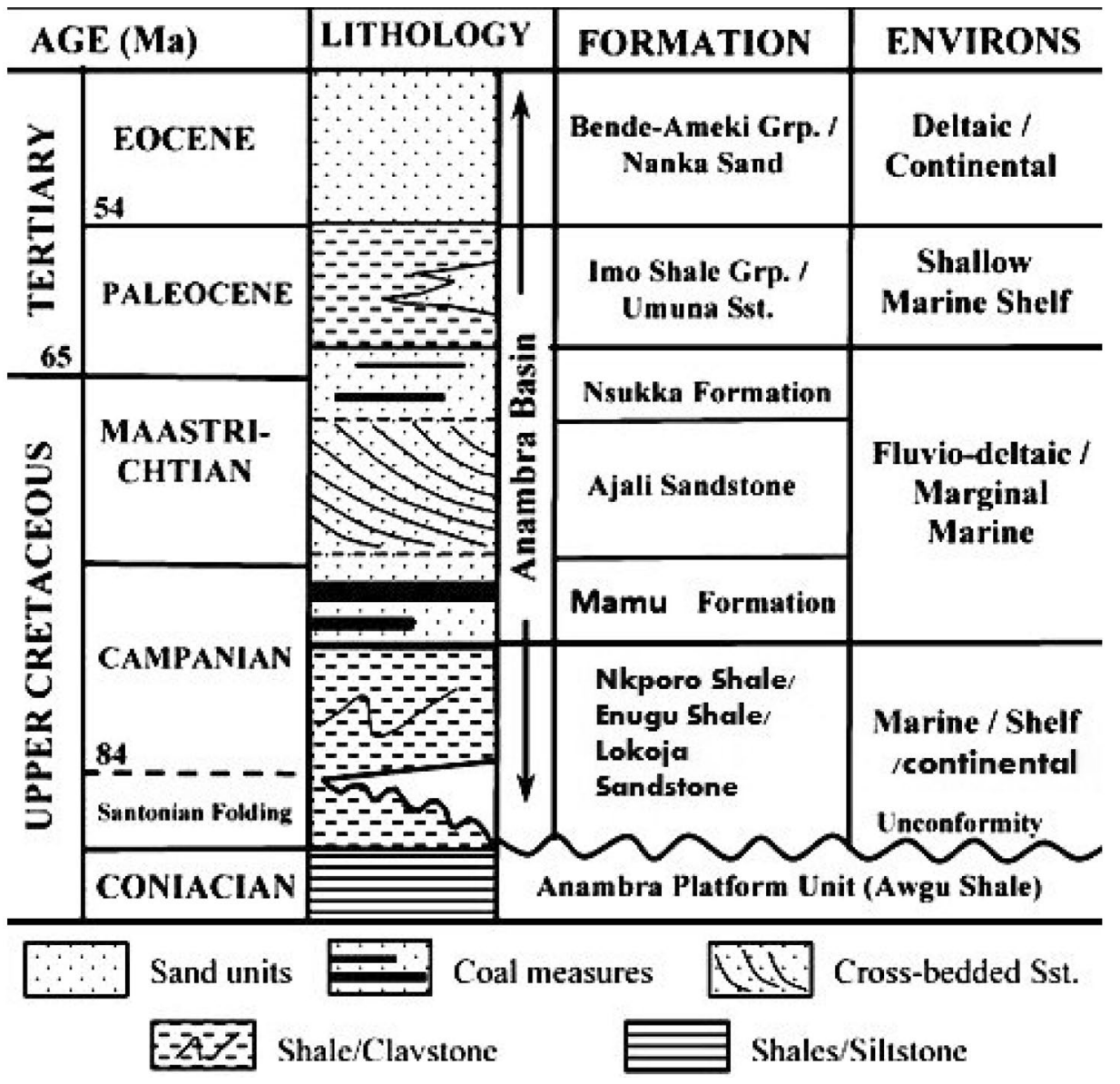

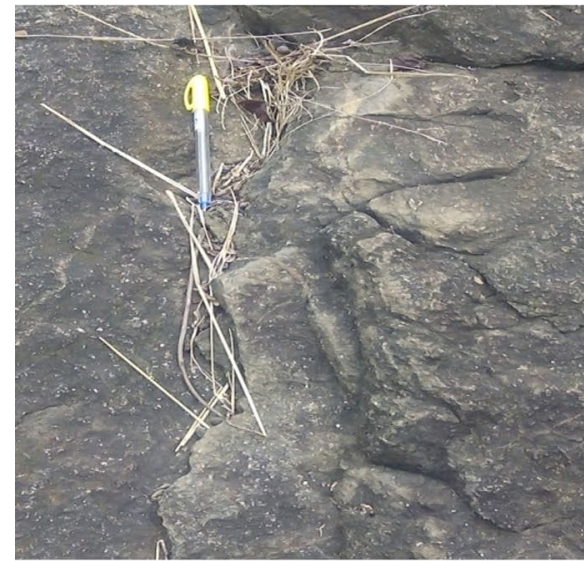

(a)

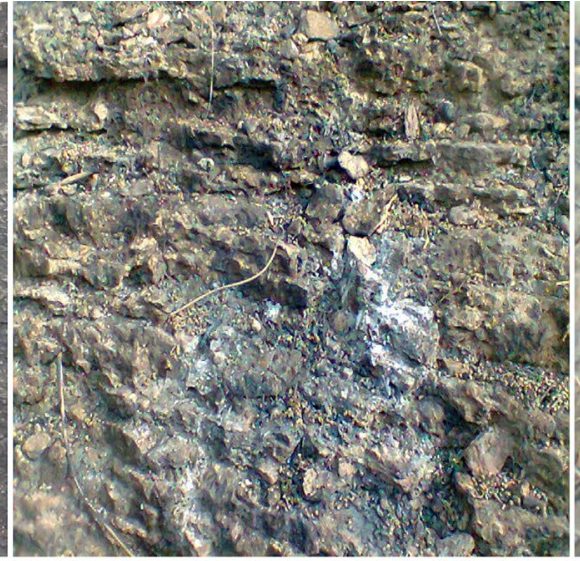

(b)

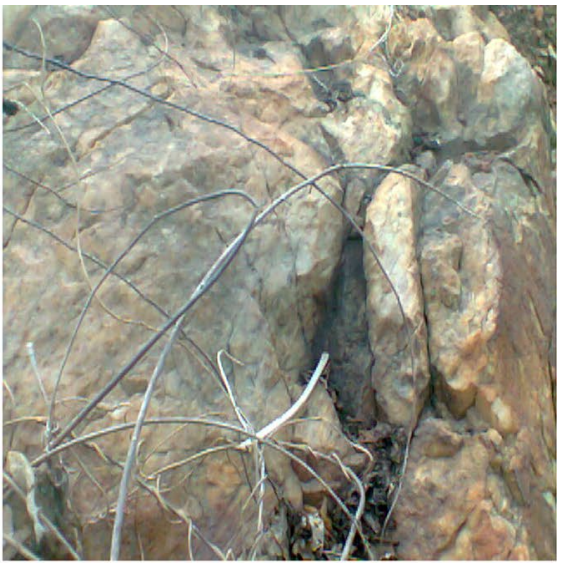

(c)

Fig.5 Sections of a porphyritic granite, $\mathbf{b}$ migmatite-gneiss and $\mathbf{c}$ quartzite (metasediment)

every location, the name of the locality, the geographical coordinates, the strike and dip of the beds at outcrop as well as the physical characteristics of the sediments visible at outcrop were determined and recorded. The field observations of outcrops at 5 different locations within Auchi and Igarra are provided in Table 1. 


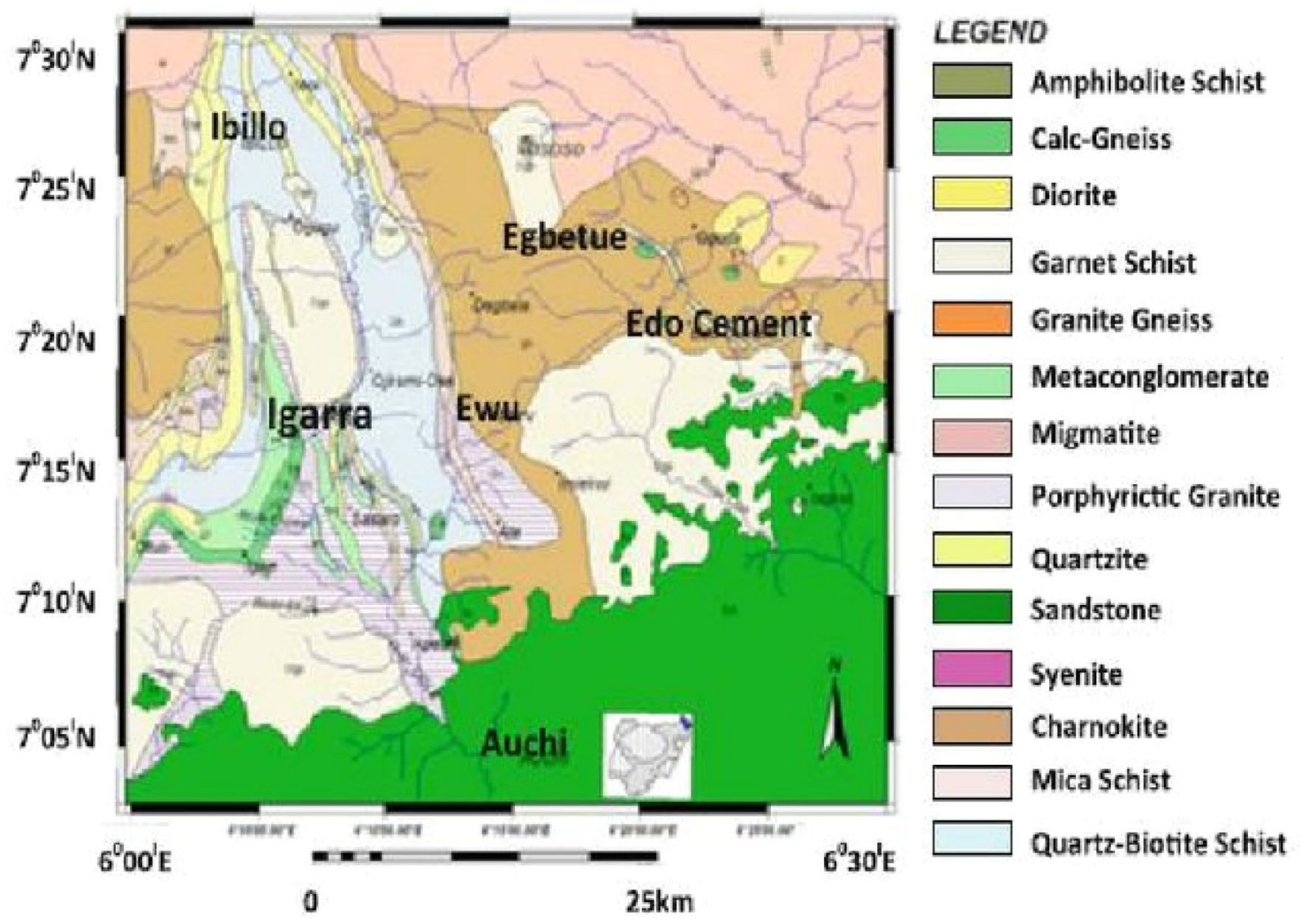

Fig.6 Geologic map of Igarra area (after Anifowose et al. 2006)

\section{Electromagnetic surveying}

In carrying out the electromagnetic survey along the profile of interest (profile X shown in Fig. 1), the very lowfrequency (VLF) method of electromagnetic surveying which involves a stationary source and a moving receiver was employed. The name of the equipment used as receiver is the ABEM WADI EM receiver. The equipment has an antenna unit which contains horizontal and vertical bars. These bars contain the transmitting and the receiving coils. The horizontal bar measured horizontal variations while the vertical bar measured vertical variations in the subsurface. The power supply unit was used to power the antenna and the receiver, while the receiver unit received the resultant field. The starting point of the survey was at about $120 \mathrm{~m}$ from SMAC Quarry Mills, along Auchi-Igarra-Ibillo road, Ikpeshi-Igarra, Akoko-Edo LGA and was run toward Auchi. A total of 155 data points was covered, and data were collected every $20 \mathrm{~m}$ along the profile. At every data point, the raw real (\%), the filtered real (\%), the raw imaginary (\%) and the filtered imaginary $(\%)$ values were read and recorded.

The VLF electromagnetic data consists of two separate components: the raw real otherwise called the in-phase data and the raw imaginary otherwise known as the quadrature data. These carry very important complex information about the geology of the subsurface of that environment. Because of the complexity of the information, it cannot be seamlessly related to the body causing the anomaly. In addition, these data carry high noise. To correct for these, filtering operators are applied, among other things, to reduce the nonlinear and harmonic noise, to transform the raw real into current density and to adequately position the amplitude of the anomalous body over its source. Hence, the filtered real and filtered imaginary data are respectively the raw real and raw imaginary data which have undergone filtering to correct the defects mentioned above. Microsoft Excel software was used to plot the survey data. The acquired survey data are shown in Table 2. 


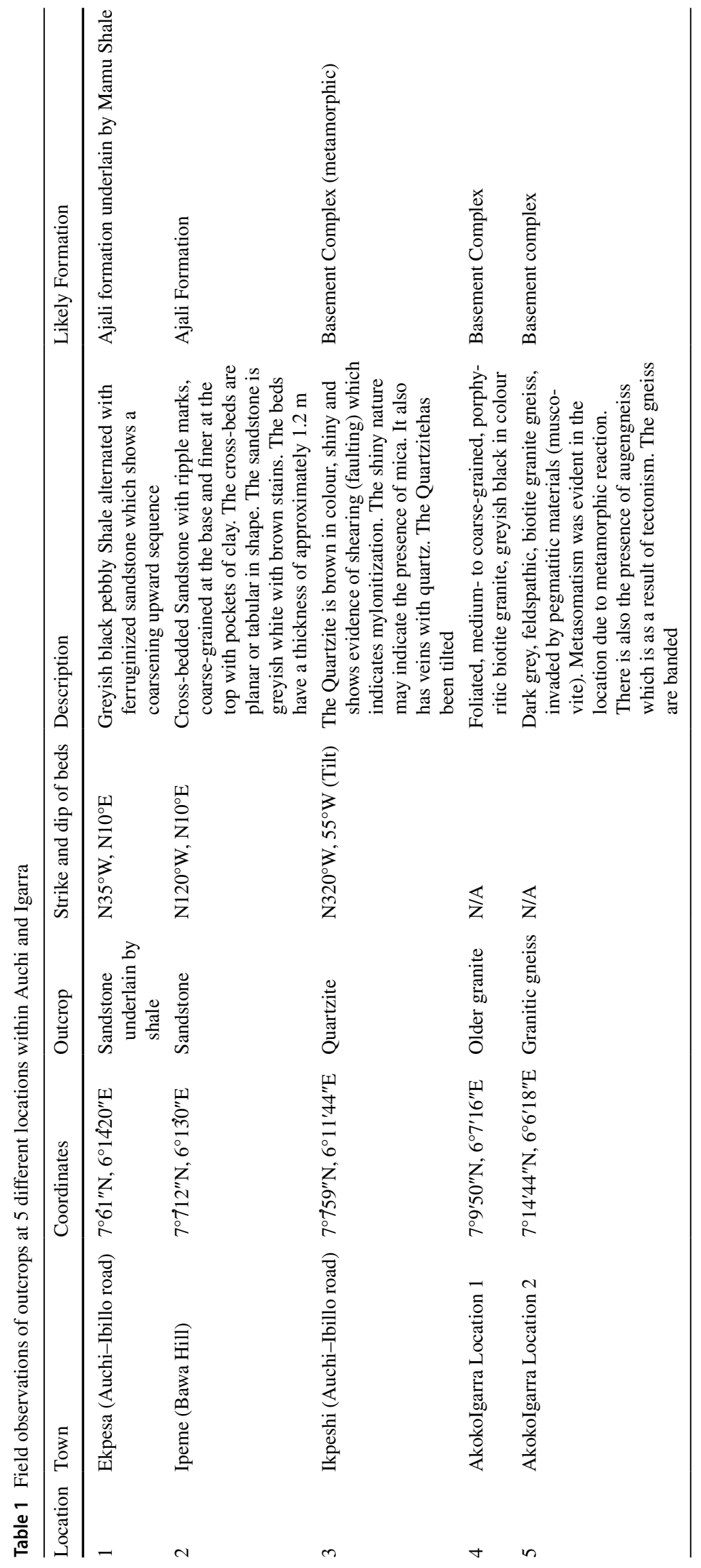


Table 2 Electromagnetic survey data acquired along Profile $\mathrm{X}$, Auchi-Igarra-Ibillo road
Electromagnetic survey data

Location: Along Auchi-Igarra-Ibillo express way

Starting point: $120 \mathrm{~m}$ from SMAC Quarry Mills, Ekpeshi Igarra, Akoko-Edo LGA, Edo State (moving toward Auchi from Igarra)

\begin{tabular}{llllll}
\hline Station (m) & Raw real (\%) & Filtered real (\%) & $\begin{array}{l}\text { Raw } \\
\text { imagi- } \\
\text { nary (\%) }\end{array}$ & $\begin{array}{l}\text { Filtered } \\
\text { imaginary } \\
(\%)\end{array}$ & Remark \\
\hline 0 & 0 & 1 & 17.5 & 0.9 & Basement
\end{tabular}

$\begin{array}{llllll}0 & 0 & 1 & 17.5 & 0.9 & \text { Basement } \\ 20 & 1.5 & -0.2 & 13.5 & -0.6 & \text { Basement } \\ 40 & -2.1 & -0.3 & 18.9 & -1.3 & \text { Basement } \\ 60 & 2.4 & -0.3 & 20.9 & 2.5 & \text { Basement } \\ 80 & -4.1 & 2 & 8.6 & 1 & \text { Basement }\end{array}$

$\begin{array}{llllll}100 & 6.7 & 4.6 & 16.8 & -1.7 & \text { Basement }\end{array}$

$\begin{array}{llllll}120 & -0.8 & -3 & 15.2 & -0.2 & \text { Basement }\end{array}$

$\begin{array}{llllll}140 & -1.2 & -2.3 & 19.7 & 0.7 & \text { Basement }\end{array}$

$\begin{array}{llllll}160 & -2.2 & -1 & 9.7 & -0.5 & \text { Basement }\end{array}$

$\begin{array}{llllll}180 & 0.5 & 1 & 19.8 & -3.6 & \text { Basement }\end{array}$

$\begin{array}{llllll}200 & -0.1 & 1.3 & 21.8 & -1 & \text { Basement }\end{array}$

$\begin{array}{llllll}220 & 2.5 & -2.2 & 24.1 & 1.7 & \text { Basement }\end{array}$

$\begin{array}{llllll}240 & -4.3 & -4.2 & 14.6 & 2.4 & \text { Basement }\end{array}$

$\begin{array}{llllll}260 & -0.8 & 0.8 & 13.8 & -1.9 & \text { Basement }\end{array}$

$\begin{array}{llllll}280 & -0.1 & 4.4 & 22.8 & -1.4 & \text { Basement }\end{array}$

$\begin{array}{llllll}300 & 4 & 6.6 & 19.9 & 1.1 & \text { Basement }\end{array}$

$\begin{array}{lll}320 & 3.7 & 1.9\end{array}$

$$
0.7
$$$$
1.9
$$

$-4.5$

$-5.4$

$-2.9$

1.5

0.8

1

$-2.1$

$-2.6$

$-2.7$

$-2.6$

0.1

$-3.5$

$-1.6$

$18.3 \quad 1$

$13.9-0.9$

Basement

1.3

22.1

$-1.7$

Basement

$20.6 \quad 0.3 \quad$ Basement

$19.2-1 \quad$ Basement

$22.8 \quad-0.8 \quad$ Basement

$21.4 \quad-0.2 \quad$ Basement

500

$-0.4$

23.9

1.5

Basement

$17 \quad 3.2$

$12-0.2$

Basement

18.4

22.4

$-2.5$

Basement

$-0.6$

Basement

$4.5 \quad 1.1$

$-0.6 \quad-0.8$

$16.5 \quad 0.3$

Basement

13.9

$-1.6$

Basement

$\begin{array}{ll}1.1 & 0.5\end{array}$

20.4

$-0.3$

Basement

$\begin{array}{ll}1.8 & 3.9\end{array}$

19.5

$-0.4$

Basement

$5.9 \quad 2.1$

20.5

0.8

Basement

$20.8 \quad 3.4$

$-5.4 \quad-5.9$

$4.1-1.1$

Basement

$4.2-25.3$

28.7

0.6

Basement

17.6

14.4

$-4.6$

Basement

Basement

Basement

Basement 
Table 2 (continued)

Electromagnetic survey data

Location: Along Auchi-Igarra-Ibillo express way

Starting point: 120 m from SMAC Quarry Mills, Ekpeshi Igarra, Akoko-Edo LGA, Edo State (moving toward Auchi from Igarra)

\begin{tabular}{llllll}
\hline Station (m) & Raw real (\%) & Filtered real (\%) & $\begin{array}{l}\text { Raw } \\
\text { imagi- } \\
\text { nary (\%) }\end{array}$ & $\begin{array}{l}\text { Filtered } \\
\text { imaginary } \\
(\%)\end{array}$ & Remark \\
\hline 880 & 4.4 & 9.3 & 21.8 & -3.4 & Basement
\end{tabular}

\begin{tabular}{|c|c|c|c|c|c|}
\hline 880 & 4.4 & 9.3 & 21.8 & -3.4 & Basement \\
\hline 900 & 8.3 & 2.4 & 21.2 & -1.2 & Basement \\
\hline 920 & 0.9 & -25.8 & 27.7 & 5.2 & Basement \\
\hline 940 & -24.5 & -30.3 & 2.2 & 5.5 & Basement \\
\hline 960 & -12.2 & -9.7 & 9.7 & 1.9 & Basement \\
\hline 980 & -2.3 & -3 & 7.8 & 9.7 & Basement \\
\hline 1000 & -3.1 & 6.2 & -23.8 & 1.7 & Basement \\
\hline 1020 & 9.1 & 10.5 & 2.2 & -5.1 & Basement \\
\hline 1040 & 1.9 & 5.1 & 0.1 & 1.8 & Basement \\
\hline 1060 & 6.1 & 1.6 & -3.9 & 0.7 & Basement \\
\hline 1080 & -5.4 & 4.1 & -6.4 & 0.1 & Basement \\
\hline 1100 & 8 & 10.4 & -3.6 & -1.4 & Basement \\
\hline 1120 & 4.2 & -0.1 & -1.1 & -0.9 & Basement \\
\hline 1140 & -2 & -3.9 & -0.8 & 0.2 & Basement \\
\hline 1160 & -1.3 & -6.1 & -3.2 & 0.1 & Basement \\
\hline 1180 & -5.2 & -10.1 & -2.2 & -0.8 & Basement \\
\hline 1200 & -3.3 & -14.6 & -0.5 & 1.3 & Basement \\
\hline 1220 & -2.1 & -11.5 & -0.6 & 7.3 & Basement \\
\hline 1240 & -1 & -1.1 & -25 & 5.6 & Basement \\
\hline 1260 & -3.5 & 5.1 & -20 & -2 & Basement \\
\hline 1280 & 8.1 & 9.5 & -12.6 & -0.4 & Basement \\
\hline 1300 & 2.8 & 2.6 & -15.9 & 0.8 & Basement \\
\hline 1320 & 2.2 & -1.3 & -16.9 & 1 & Basement \\
\hline 1340 & -3.6 & -1.9 & -19.7 & 0.9 & Basement \\
\hline 1360 & 0.9 & -0.4 & -19 & 1.8 & Basement \\
\hline 1380 & -0.6 & -2.2 & -19.2 & 5.3 & Basement \\
\hline 1400 & -4.2 & 5.8 & -44.1 & -2.9 & Basement \\
\hline 1420 & 11 & 3 & -4.5 & -3.6 & Basement \\
\hline 1440 & -7.2 & 0.2 & -31.2 & 1.2 & Basement \\
\hline 1460 & 5 & 12.9 & -14.4 & -5.7 & Basement \\
\hline 1480 & 9.2 & 0.5 & -10.1 & 5.3 & Basement \\
\hline 1500 & -4.5 & -9.7 & -27.4 & 7.6 & Basement \\
\hline 1520 & -5.8 & -2.9 & -41.8 & -2.1 & Basement \\
\hline 1540 & -0.6 & 2.7 & -22.3 & -11.1 & Basement \\
\hline 1560 & 4.4 & -2.5 & -2.7 & -5.9 & Basement \\
\hline 1580 & -5.9 & -3.6 & 2.1 & 6.6 & Basement \\
\hline 1600 & 0.5 & 0.7 & -35 & 5.3 & Basement \\
\hline 1620 & 2.9 & -0.4 & -15.4 & -1.3 & Basement \\
\hline 1640 & -14.8 & 3.1 & -30.3 & -4.2 & Basement \\
\hline 1660 & 17.2 & 5 & -0.8 & -7.7 & Basement \\
\hline 1680 & -8.2 & -13.5 & -2.8 & 4.3 & Basement \\
\hline 1700 & -4.5 & -4.5 & -19.1 & 4.7 & Basement \\
\hline 1720 & -2.4 & -4.4 & -16.8 & 6.4 & Basement \\
\hline 1740 & -2.8 & -2 & -39.1 & 0.3 & Basement \\
\hline
\end{tabular}


Table 2 (continued)

\begin{tabular}{|c|c|c|c|c|c|}
\hline \multicolumn{6}{|c|}{ Electromagnetic survey data } \\
\hline \multicolumn{6}{|c|}{ Location: Along Auchi-Igarra-Ibillo express way } \\
\hline \multicolumn{6}{|c|}{$\begin{array}{l}\text { Starting point: } 120 \mathrm{~m} \text { from SMAC Quarry Mills, Ekpeshi Igarra, Akoko-Edo LGA, Edo State (moving } \\
\text { toward Auchi from Igarra) }\end{array}$} \\
\hline Station $(\mathrm{m})$ & Raw real $(\%)$ & Filtered real $(\%)$ & $\begin{array}{l}\text { Raw } \\
\text { imagi- } \\
\text { nary }(\%)\end{array}$ & $\begin{array}{l}\text { Filtered } \\
\text { imaginary } \\
(\%)\end{array}$ & Remark \\
\hline 1760 & -0.7 & 2.8 & -21.6 & -11.7 & Basement \\
\hline 1780 & 4.4 & -1.1 & 4.1 & -3.7 & Basement \\
\hline 1800 & -5.1 & -1.6 & -6.9 & 6.5 & Basement \\
\hline 1820 & 1.8 & 7.5 & -24.6 & 4.2 & Basement \\
\hline 1840 & 4 & 9.5 & -25.3 & -3.2 & Basement \\
\hline 1860 & 6.6 & 7.4 & -8.9 & -3.8 & Basement \\
\hline 1880 & 2.2 & 3.4 & -9.9 & -8.1 & Basement \\
\hline 1900 & 0.4 & 3.7 & -13.7 & -1.4 & Basement \\
\hline 1920 & 5.4 & -4.1 & -7.5 & -1.5 & Basement \\
\hline 1940 & -8.6 & -6.9 & -10.4 & -2.1 & Basement \\
\hline 1960 & -0.7 & 2.7 & -1 & -1.6 & Basement \\
\hline 1980 & 3.1 & -0.2 & -5.9 & 0.7 & Basement \\
\hline 2000 & -4 & 3.1 & -6.1 & -1.8 & Suspected termination of basement \\
\hline 2020 & 7.3 & 4.8 & -1.5 & -1.7 & Suspected boundary \\
\hline 2040 & -2.1 & -0.7 & 1.4 & 1.2 & Suspected boundary \\
\hline 2060 & 2.8 & -0.6 & -6.3 & 2.4 & Suspected boundary \\
\hline 2080 & -4 & 0.3 & -6.6 & 0.6 & Suspected boundary \\
\hline 2100 & 2 & 8.2 & -10.2 & -2.2 & Suspected boundary \\
\hline 2120 & 7.2 & 2 & 4.2 & -0.3 & Suspected beginning of sedimentary \\
\hline 2140 & -3.3 & -5.3 & -9 & 3 & Sedimentary \\
\hline 2160 & 0.1 & -3.1 & -7.7 & -1.2 & Sedimentary \\
\hline 2180 & -10.5 & 19 & -8.2 & -4.6 & Sedimentary \\
\hline 2200 & 26.5 & 26.3 & 6.6 & -6.2 & Sedimentary \\
\hline 2220 & 8.7 & -10.6 & 13.6 & -0.6 & Sedimentary \\
\hline 2240 & -12.8 & -19.7 & 1.4 & 2.8 & Sedimentary \\
\hline 2260 & -3.5 & -16.4 & 0.6 & 0.4 & Sedimentary \\
\hline 2280 & -12.6 & -7.4 & 1.8 & 0.5 & Sedimentary \\
\hline 2300 & 0.3 & 11.1 & 0.9 & 0.1 & Sedimentary \\
\hline 2320 & 8.9 & 16.6 & 1 & -0.3 & Sedimentary \\
\hline 2340 & 8 & 18.2 & 1.9 & -0.2 & Sedimentary \\
\hline 2360 & 13.1 & 12.4 & 1.3 & -0.2 & Sedimentary \\
\hline 2380 & 2.2 & -0.1 & 2.6 & -0.6 & Sedimentary \\
\hline 2400 & 2.2 & -16.8 & 1 & -1.6 & Sedimentary \\
\hline 2420 & -13.9 & -27.4 & 10.2 & 0.6 & Sedimentary \\
\hline 2440 & -26 & 16.2 & -2.8 & 0.6 & Sedimentary \\
\hline 2460 & 34.8 & 25.8 & 6.2 & -2.5 & Sedimentary \\
\hline 2480 & 5.5 & -32.1 & 7.3 & 1 & Sedimentary \\
\hline 2500 & -32.5 & -27.1 & 3.1 & 0.7 & Sedimentary \\
\hline 2520 & -2.4 & -4.7 & 2.4 & -0.8 & Sedimentary \\
\hline 2540 & -9.8 & 6 & 7 & 0.7 & Sedimentary \\
\hline 2560 & 3.8 & 34.2 & 3.5 & 4.2 & Sedimentary \\
\hline 2580 & 28.9 & 21.6 & -7.5 & 2.6 & Sedimentary \\
\hline 2600 & -2.7 & 10.6 & -5.5 & -1.4 & Sedimentary \\
\hline 2620 & 14.7 & 8.1 & -1.3 & -3 & Sedimentary \\
\hline
\end{tabular}


Table 2 (continued)

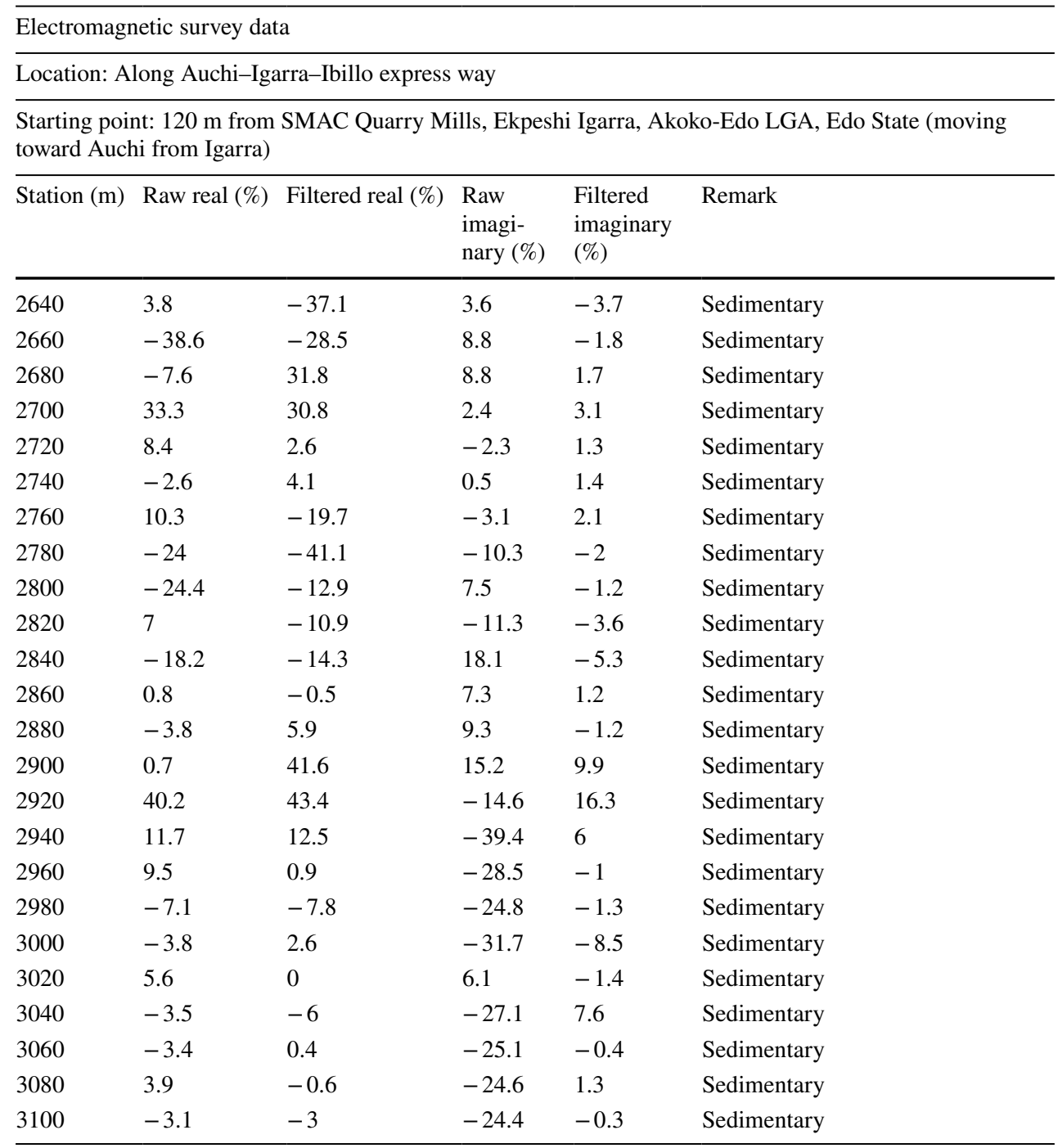

\section{Magnetic surveying}

In carrying out the magnetic survey along the same profile $X$, a set of field equipment which includes the magnetometer and the sensor unit was employed. As in electromagnetic survey, the starting point of the ground magnetic survey was at about $120 \mathrm{~m}$ from SMAC Quarry Mills, along Auchi-Igarra-Ibillo road, Ikpeshi-Igarra, Akoko-Edo LGA and was run toward Auchi. A total of 44 data points was covered, and data were collected every $50 \mathrm{~m}$ for the first $1 \mathrm{~km}$ and every $100 \mathrm{~m}$ for the remaining $2.3 \mathrm{~km}$ along the profile. At every data point, the peak value $(\mu \mathrm{T})$ was read and recorded twice, and the field value $(\mu \mathrm{T})$ was also read and recorded twice. Their average values were later calculated. This was done to reduce errors in the readings. Also, the time at which data were acquired at every location was read and recorded.

Microsoft Excel software was used to plot the survey data. The acquired survey data are shown in Table 3.

\section{Results}

The results of the geologic field investigations of outcrops carried out at five different locations within Auchi and Igarra areas are presented below. 
Table 3 Ground magnetic survey data acquired along Profile X, Auchi-Igarra-Ibillo road

\begin{tabular}{|c|c|c|c|c|c|c|c|}
\hline Distance (m) & Peak 1 and $2(\mu \mathrm{T})$ & Average peak $(\mu \mathrm{T})$ & $\begin{array}{l}\text { Variation in } \\
\text { peak }(\mu \mathrm{T})\end{array}$ & Field 1 and $2(\mu \mathrm{T})$ & Average field $(\mu \mathrm{T})$ & $\begin{array}{l}\text { Variation in } \\
\text { field }(\mu \mathrm{T})\end{array}$ & Time (hours) \\
\hline 0 & $25.585,22.008$ & 23.797 & 0.000 & $21.550,21.639$ & 21.595 & 0.000 & $14: 31$ \\
\hline 50 & $16.601,18.200$ & 17.401 & -6.396 & $16.343,15.007$ & 15.675 & -5.920 & $14: 35$ \\
\hline 100 & $16.066,15.977$ & 16.022 & -1.379 & $15.781,15.821$ & 15.801 & 0.126 & $14: 42$ \\
\hline 150 & $14.177,13.764$ & 13.971 & -2.051 & $13.848,13.736$ & 13.792 & -2.009 & $14: 44$ \\
\hline 200 & $15.158,14.738$ & 14.948 & 0.977 & $14.583,14.681$ & 14.632 & 0.840 & $14: 46$ \\
\hline 250 & $17.236,17.214$ & 17.225 & 2.277 & $17.214,17.20$ & 17.212 & 2.580 & $14: 49$ \\
\hline 300 & $18.431,17.815$ & 18.123 & 0.898 & $17.544,17.581$ & 17.563 & 0.351 & $14: 53$ \\
\hline 350 & $15.721,15.988$ & 15.855 & -2.268 & $15.753,15.335$ & 15.544 & -2.019 & $14: 56$ \\
\hline 400 & $15.175,15.114$ & 15.145 & -0.71 & $14.758,15.006$ & 14.882 & -0.662 & $14: 58$ \\
\hline 450 & $15.466,15.290$ & 15.378 & 0.233 & $15.221,15.281$ & 15.251 & 0.369 & $15: 00$ \\
\hline 500 & $14.496,14.496$ & 14.496 & -0.882 & $14.011,14.011$ & 14.011 & -1.240 & $15: 03$ \\
\hline 550 & $15.958,15.958$ & 15.958 & 1.462 & $15.951,15.951$ & 15.951 & 1.940 & $15: 05$ \\
\hline 600 & $16.931,16.738$ & 16.835 & 0.877 & $16.669,16.624$ & 16.647 & 0.696 & $15: 08$ \\
\hline 650 & $16.370,16.414$ & 16.392 & -0.443 & $16.222,16.211$ & 16.217 & -0.430 & $15: 09$ \\
\hline 700 & $17.480,18.480$ & 17.98 & 1.588 & $18.358,17.840$ & 18.099 & 1.882 & $15: 12$ \\
\hline 750 & $15.106,15.131$ & 15.119 & -2.861 & $15.054,14.880$ & 14.967 & -3.132 & $15: 14$ \\
\hline 800 & $15.495,15.380$ & 15.438 & 0.319 & $14.988,15.347$ & 15.168 & 0.201 & $15: 16$ \\
\hline 850 & $14.513,14.193$ & 14.353 & -1.085 & $14.162,14.173$ & 14.168 & -1.000 & $15: 18$ \\
\hline 900 & $14.899,14.953$ & 14.926 & 0.573 & $14.776,14.788$ & 14.782 & 0.614 & $15: 19: 00$ \\
\hline 950 & $15.500,15.381$ & 15.441 & 0.515 & $15.310,15.351$ & 15.331 & 0.549 & $15: 22$ \\
\hline 1000 & $16.616,16.597$ & 16.607 & 1.166 & $16.600,16.588$ & 16.594 & 1.263 & $15: 24$ \\
\hline 1100 & $15.000,14.613$ & 14.807 & -1.8 & $14.259,14.155$ & 14.207 & -2.387 & $15: 43$ \\
\hline 1200 & $11.781,11.789$ & 11.785 & -3.022 & $11.750,11.779$ & 11.765 & -2.442 & $15: 46$ \\
\hline 1300 & $13.571,14.741$ & 14.156 & 2.371 & $13.481,13.500$ & 13.491 & 1.726 & $15: 49$ \\
\hline 1400 & $11.795,11.658$ & 11.727 & -2.429 & $11.765,11.622$ & 11.694 & -1.797 & $15: 52$ \\
\hline 1500 & $13.667,13.662$ & 13.665 & 1.938 & $13.640,13.648$ & 13.644 & 1.950 & $15: 54$ \\
\hline 1600 & $13.325,13.282$ & 13.304 & -0.361 & $13.268,13.263$ & 13.266 & -0.378 & $15: 57$ \\
\hline 1700 & $14.171,13.358$ & 13.765 & 0.461 & $14.331,13.341$ & 13.836 & 0.570 & $16: 00$ \\
\hline 1800 & $13.324,13.342$ & 13.333 & -0.432 & $13.313,13.335$ & 13.324 & -0.512 & $16: 03$ \\
\hline 1900 & $13.609,13.563$ & 13.586 & 0.253 & $13.534,13.552$ & 13.543 & 0.219 & 16:06 \\
\hline 2000 & $14.566,14.441$ & 14.504 & 0.918 & $14.432,14.437$ & 14.435 & 0.892 & $16: 09$ \\
\hline 2100 & $15.497,14.750$ & 15.124 & 0.62 & $15.280,14.720$ & 15 & 0.565 & $16: 13$ \\
\hline 2200 & $12.495,12.468$ & 12.482 & -2.642 & $12.397,12.349$ & 12.373 & -2.627 & $16: 16$ \\
\hline 2300 & $9.077,9.278$ & 9.178 & -3.304 & $8.847,8.925$ & 8.886 & -3.487 & $16: 19$ \\
\hline 2400 & $10.419,10.249$ & 10.334 & 1.156 & $10.142,10.216$ & 10.179 & 1.293 & $16: 23$ \\
\hline 2500 & $11.677,11.676$ & 11.677 & 1.343 & $11.560,11.616$ & 11.588 & 1.409 & $16: 27$ \\
\hline 2600 & $13.097,13.270$ & 13.184 & 1.507 & $13.018,13.030$ & 13.024 & 1.436 & $16: 31$ \\
\hline 2700 & $13.745,13.478$ & 13.612 & 0.428 & $13.685,13.427$ & 13.556 & 0.532 & $16: 35$ \\
\hline 2800 & $13.717,13.702$ & 13.71 & 0.098 & $13.680,13.618$ & 13.694 & 0.093 & $16: 38$ \\
\hline 2900 & $14.641,14.641$ & 14.641 & 0.931 & $14.270,13.200$ & 13.735 & 0.086 & $16: 41$ \\
\hline 3000 & $15.561,15.111$ & 15.336 & 0.695 & $15.007,14.657$ & 14.832 & 1.097 & $16: 44$ \\
\hline 3100 & $15.081,14.335$ & 14.708 & -0.628 & $14.244,14.257$ & 14.251 & -0.581 & $16: 47$ \\
\hline 3200 & $10.043,10.137$ & 10.09 & -4.618 & $9.886,9.700$ & 9.793 & -4.458 & $16: 51$ \\
\hline 3300 & $8.580,4.556$ & 6.568 & -3.522 & $4.419,4.256$ & 4.338 & -5.455 & $16: 54$ \\
\hline
\end{tabular}




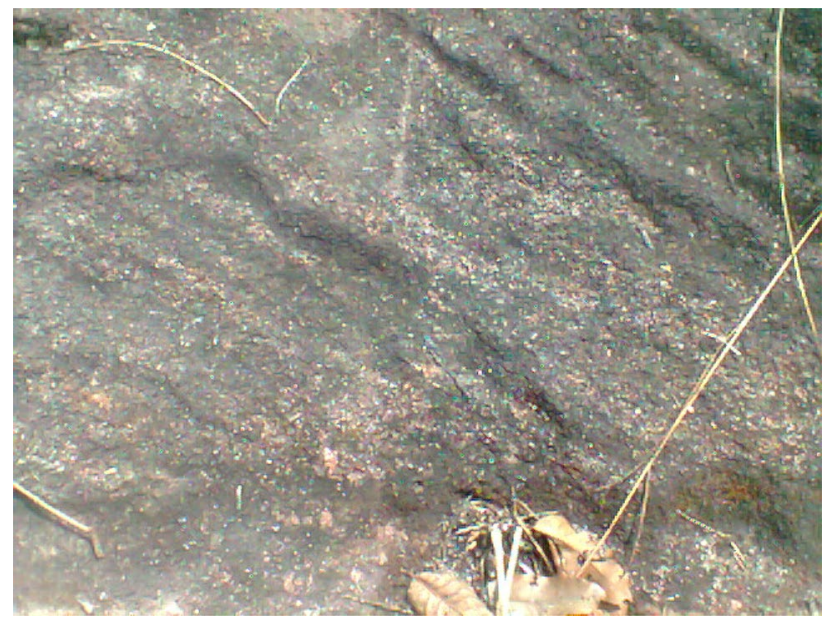

Fig.7 Vertical section of tabular, cross-bedded, fine-to-mediumgrained sandstone facie observed at location 2

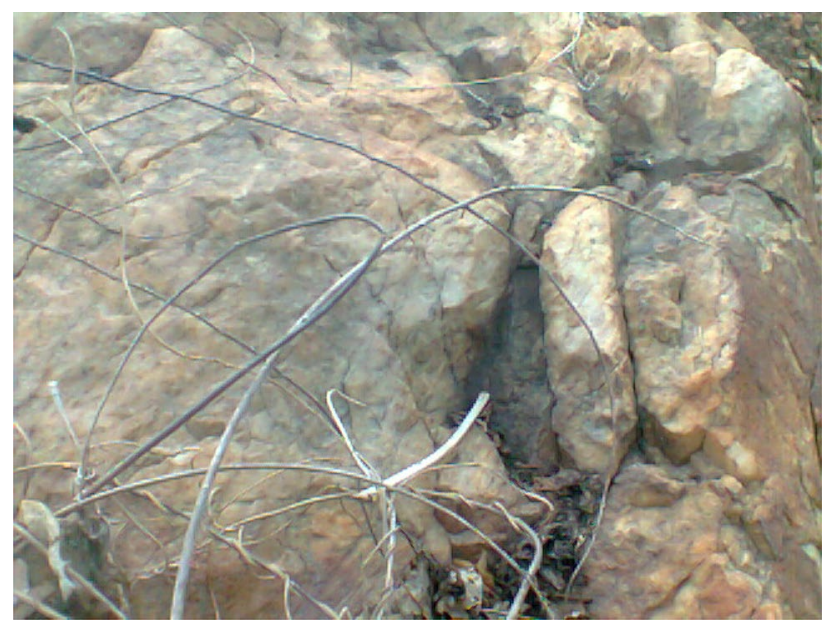

Fig.8 A section of the quartzite observed at location 3
Location 1 (Ekpesa, Auchi): At this location, an outcrop of ferruginized sandstone underlain by greyish black pebbly shale was seen. This shows a coarsening upward sequence.

Location 2 (Ipeme, Auchi): This location has an exposure of tabular cross-bedded, greyish white sandstone with ripple marks, coarse-grained at the base and finer at the top with pockets of clay. This outcrop shows a fining upward sequence (Fig. 7).

Location 3 (Ikpeshi-Igarra): Here, an outcrop of light brown, shiny quartzite was observed. By visualization, the quartzite shows evidence of shearing (faulting) which indicates mylonitization. The shiny nature may indicate the presence of mica. It also has veins with quartz. The quartzite has been tilted (Fig. 8).

Location 4 (Akoko-Igarra 1) This location is at Akoko-Igarra and it is an older granite complex. The biotite granites are foliated, medium-to-coarse-grained, porphyritic and greyish black in colour. They have areas of jointing which helps to determine their direction of weakness (Fig. 9).

Location 5 (Akoko-Igarra 2): This location has an exposure of granite gneiss. This granitic gneiss is dark grey in colour, feldspathic and invaded by pegmatitic materials (muscovite). Metasomatism was evident in the location due to metamorphic reaction. There is also the presence of augengneiss which is as a result of tectonism. The gneiss is specially banded (Figs. 10, 11).

\section{Electromagnetic survey}

The data in Table 2 were obtained from the electromagnetic survey carried out along profile $\mathrm{X}$ within the study area.

The above data were plotted using Microsoft Excel software. The filtered real (\%) and raw real (\%) were plotted against distance (metres) on the same graph (Fig. 12); the raw real (\%) was plotted against distance (Fig. 13); and finally, the filtered real (\%) was also plotted against distance (Fig. 14).

\section{Magnetic survey}

The data in Table 3 were obtained from the ground magnetic survey carried out along profile $\mathrm{X}$ within the study area.

The above data were also plotted using Microsoft Excel software. The variation in peak measured in MicroTesla $(\mu \mathrm{T})$ was plotted against distance (metres); the variation in field $(\mu \mathrm{T})$ was plotted against distance (metres), and finally, the variations in peak and field $(\mu \mathrm{T})$ were plotted against distance $(\mathrm{m})$ on the same graph. The plots are shown in Figs.15, 16 and 17 respectively. 


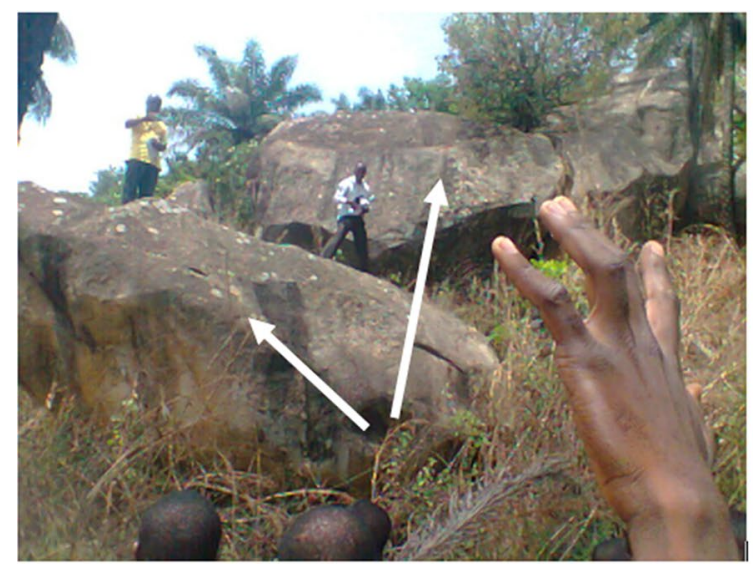

(a)

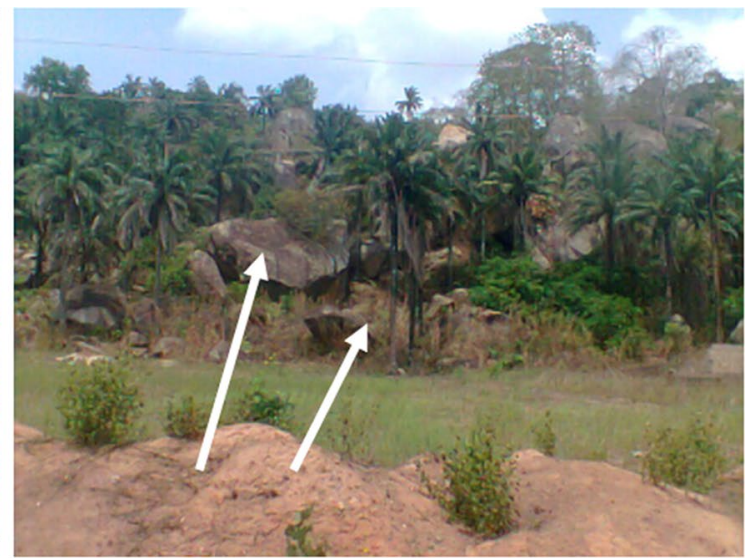

(b)

Fig.9 Sections of the foliated, porphyritic biotite granites observed at a first Akoko-Igarra location, outcrop 1, b first Akoko-Igarra location, outcrop 2. Both constitute location 4

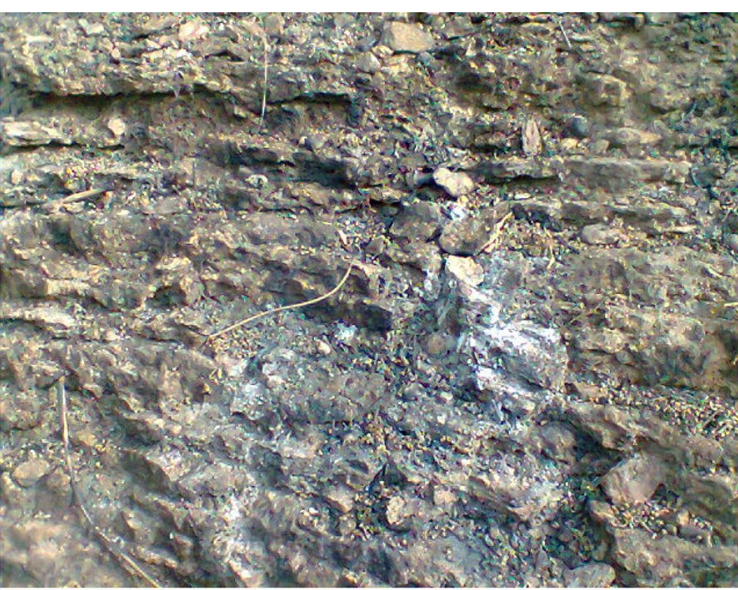

(a)

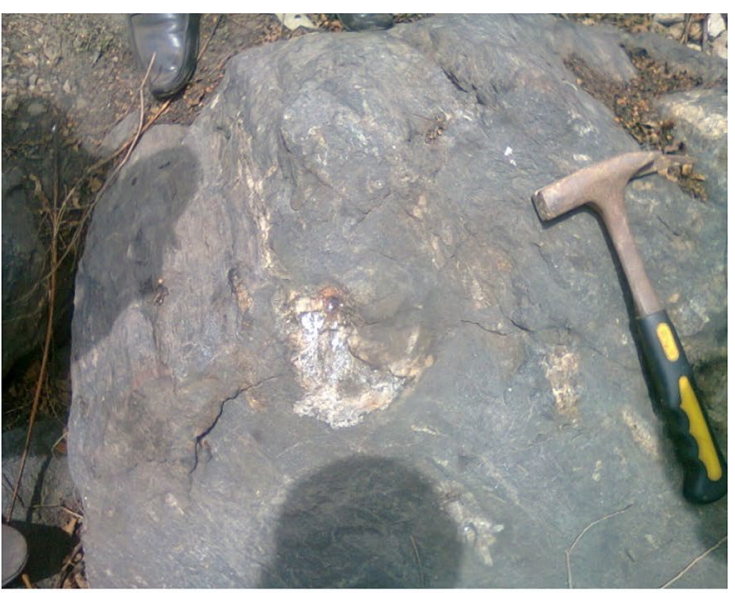

(b)

Fig.10 Sections of the a banded gneiss observed at second Akoko-Igarra location, outcrop 1, b dark grey, feldspathic, biotite granite gneiss observed at second Akoko-Igarra location, outcrop 2. Both constitute location 5

\section{Discussion}

From the field observations, three different lithofacies belonging to two lithostratigraphic units were observed within Auchi area studied (locations 1 and 2). These are: shale unit, cross-bedded sandstone unit and ferruginized sandstone unit. The shale unit is overlain by the cross-bedded sandstone and the ferruginized sandstone units which are in agreement with previous studies carried out in the area. The outcrops observed within Igarra area are rocks of the Precambrian basement complex, a metasedimentary suite which has been intruded by igneous rocks of mostly Pan-African age.

From the results of the electromagnetic survey carried out, it can be seen that from a distance of $0 \mathrm{~m}$ (starting point) to a distance of about $2000 \mathrm{~m}$, the conductivity was much lower when compared to the conductivity from a distance of about $2120 \mathrm{~m}$ up to $3000 \mathrm{~m}$. This shows that the rocks underlying the area between $0 \mathrm{~m}$ to about $2000 \mathrm{~m}$ have lower electrical conductivities when compared to the rocks underlying the area between about $2120 \mathrm{~m}$ up to $3000 \mathrm{~m}$. From theoretical knowledge, basement rocks have very low electrical conductivities because of their lack of porosity, saturation and possibly salinity, while sedimentary rocks have higher conductivities because of these properties. 


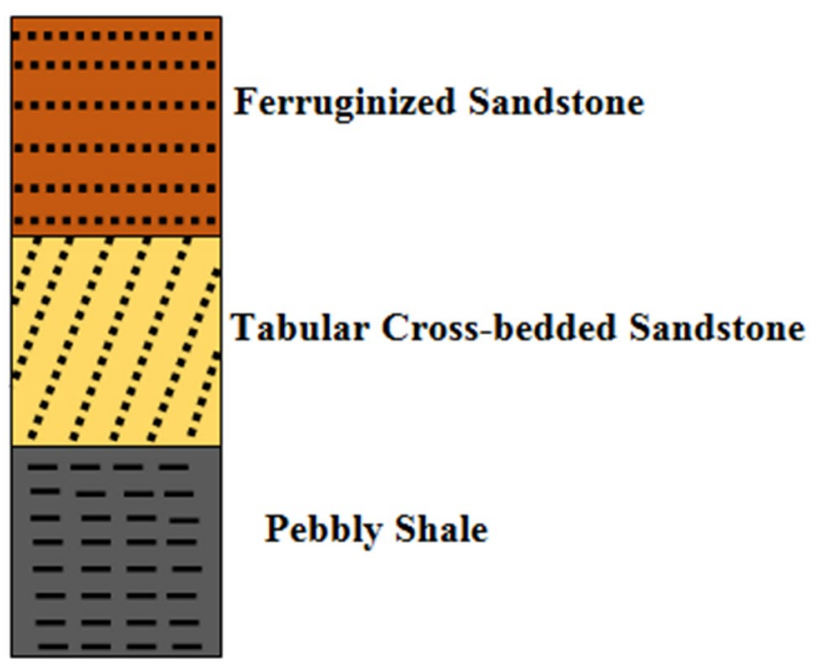

Fig.11 Observed lithofacie units of Auchi area

Hence, since the study profile is underlain by basement terrain on one section and sedimentary terrain on the other, it can be deduced from the plots that the distance between $0 \mathrm{~m}$ to about $2000 \mathrm{~m}$ is underlain by basement rocks because of their lower electrical conductivity values, while the area between a distance of about $2120 \mathrm{~m}$ up to $3000 \mathrm{~m}$ is underlain by sedimentary rocks because of their higher electrical conductivity values. Also, the area between a distance of 2000 and $2120 \mathrm{~m}$ (about $120 \mathrm{~m}$ ) can be said to be the transition zone and boundary between basement and sedimentary environments.

From the results of the ground magnetic survey carried out, variations in magnetic susceptibility are higher from a distance of $0 \mathrm{~m}$ to a distance of about $1900 \mathrm{~m}$ than from a distance of about $2100 \mathrm{~m}$ up to $3000 \mathrm{~m}$. Considering the linear variation in peak $(\mu \mathrm{T})$ and the linear variation in field $(\mu \mathrm{T})$ in the plots, it can be seen that the average magnetic susceptibility decreased when moving from a distance of $0 \mathrm{~m}$ up to a distance of $3000 \mathrm{~m}$. The magnetic susceptibility of most rocks is directly proportional to their content of ferromagnetic minerals like magnetite, ilmenite, pyrrhotite and franklinite (Kearey et al. 2002). Basement rocks contain more of these magnetic minerals than sedimentary rocks.

It can be deduced from the plots that the distance between $0 \mathrm{~m}$ to about $1900 \mathrm{~m}$ is underlain by basement rocks because of their higher magnetic susceptibility values, while the area between a distance of about $2100 \mathrm{~m}$ up to $3000 \mathrm{~m}$ is underlain by sedimentary rocks because of their lower magnetic susceptibility values. The area between a distance of $1900 \mathrm{~m}$ to $2100 \mathrm{~m}$ (about $200 \mathrm{~m}$ ) can be said to be the transition zone and boundary between basement terrain and sedimentary terrain. These are in agreement with the information obtained from the electromagnetic survey analysed above.

A schematic model of the basement-sedimentary geometry along profile $\mathrm{X}$ is shown in Fig. 18.

\section{Conclusion}

The field geologic investigation of some outcrops within Auchi and Igarra area of Edo State, Southern Nigeria, was carried out. Also, an electromagnetic and ground magnetic survey was carried out along profile $\mathrm{X}$ within Auchi-Igarra area. From the results of these geologic and geophysical investigations carried out, it can be concluded that the sediments of Auchi areas studied (locations 1 and 2) are made up of three major lithofacies: the basal shale unit, the tabular cross-bedded sandstone unit and the ferruginized sandstone unit. The shale unit is overlain by the

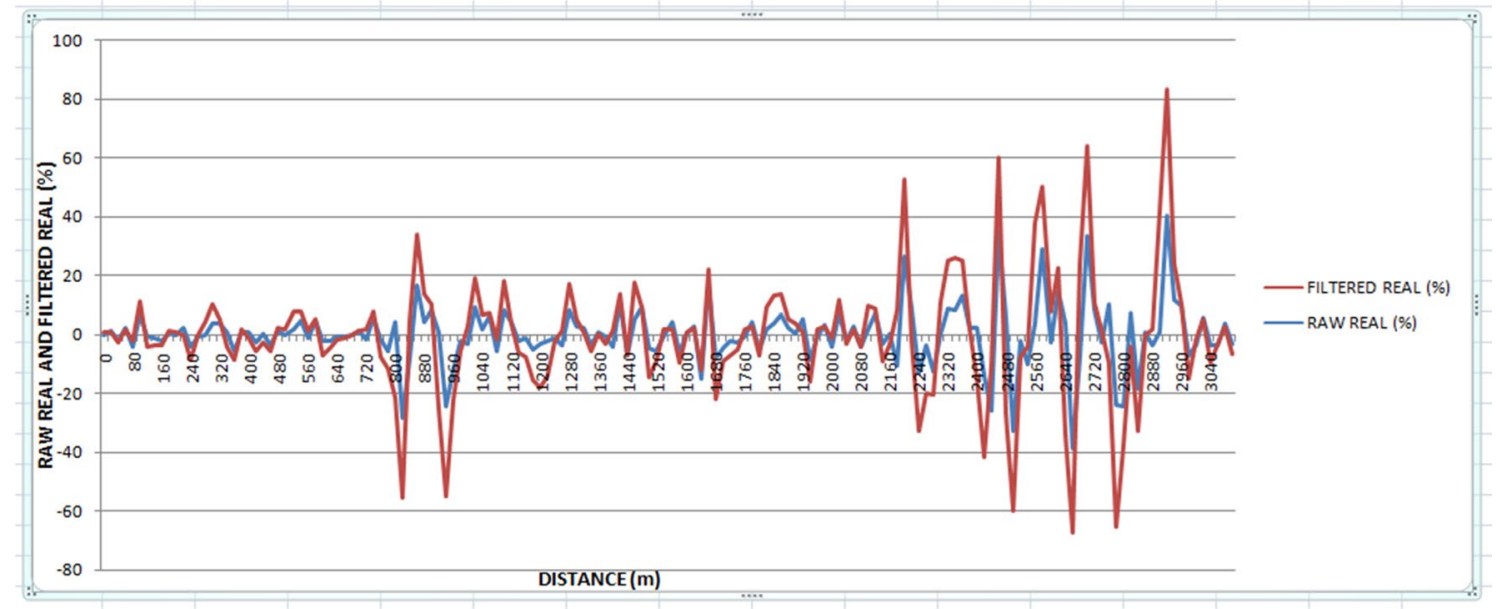

Fig.12 Plot of raw real (\%) and filtered real (\%) against distance (metres) 


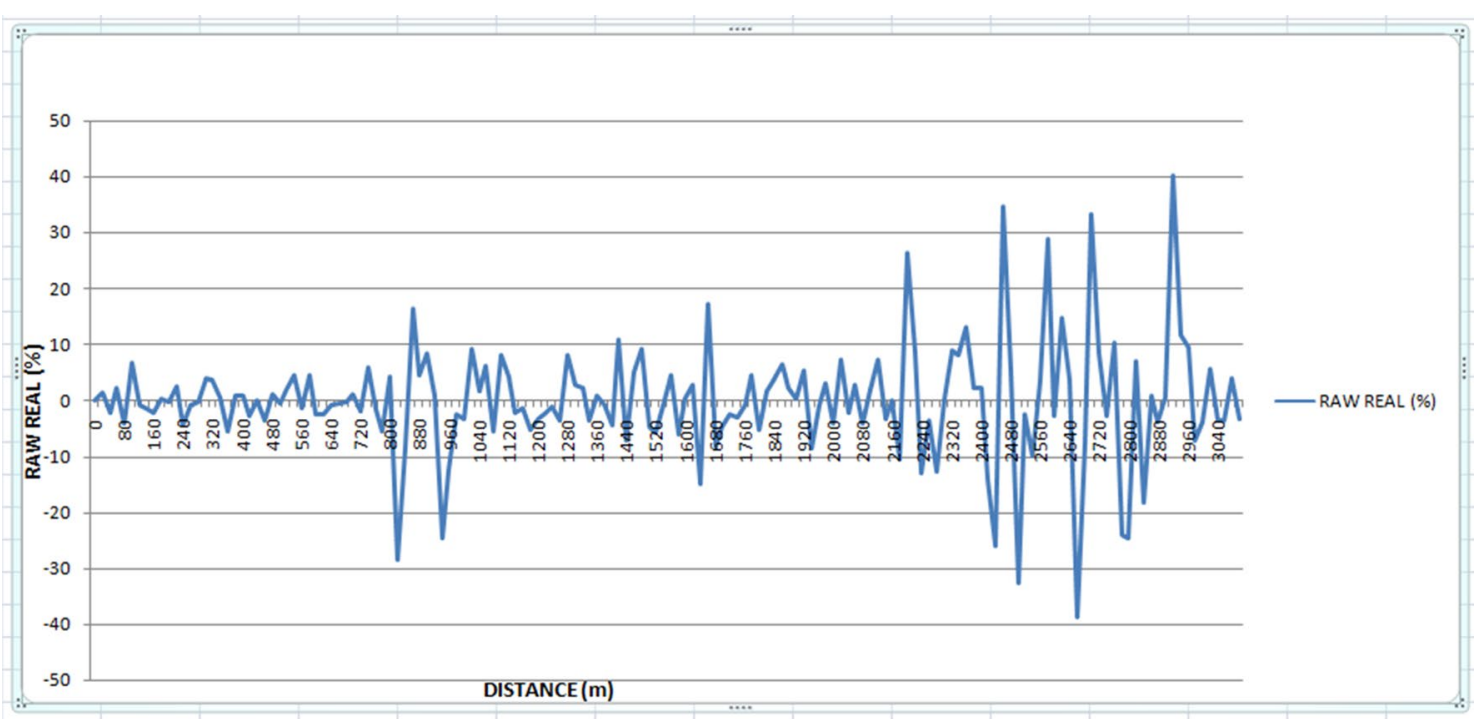

Fig.13 Plot of raw real (\%) against distance (metres)

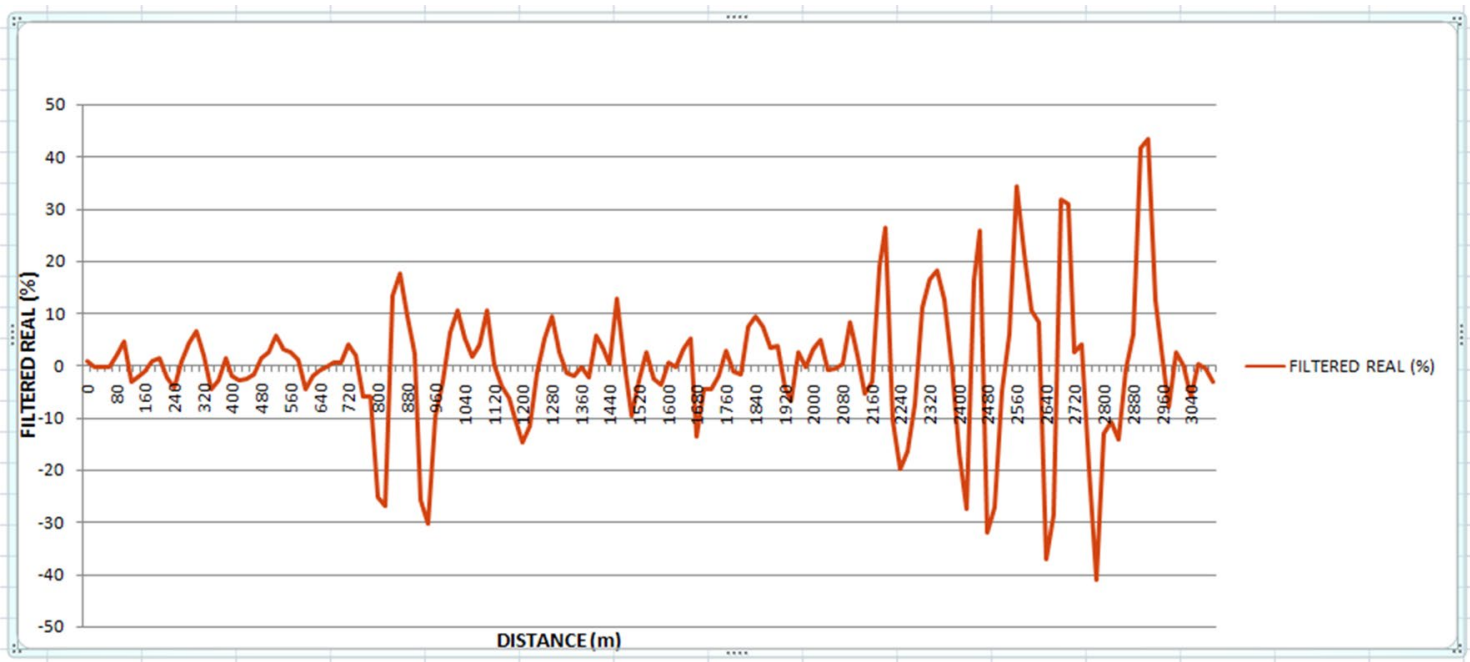

Fig.14 Plot of filtered real (\%) against distance (metres)

cross-bedded sandstone and the ferruginized sandstone units which are in agreement with previous studies carried out in the area.

Rocks of the Precambrian basement complex underlie Igarra area. The area is underlain by metasediments that have been intruded by igneous rocks. Results show the presence of three major groups of igneous and metamorphic rocks within the area and they are the migmatite-gneiss group, metasediments and porphyritic granites.

From the two geophysical surveys carried out, the boundary between basement terrain and sedimentary terrain in this area lies at approximately between a distance of 2000 and $2120 \mathrm{~m}$ from SMAC Quarry Mills, along Auchi-Igarra-Ibillo road, Ikpeshi-Igarra, Akoko-Edo Local Government Area, Edo State, Southern Nigeria (moving toward Auchi from Igarra).

From the entire investigations carried out, it is recommended that more outcrop locations should be accessed and investigated within the study area. This might give additional information about the geology of the area. In future, data or sampling points on the acquisition profile should be 
Fig.15 Plot of variation in peak $(\mu \mathrm{T})$ against distance (metres)

Fig.16 Plot of variation in field $(\mu \mathrm{T})$ against distance (metres)

Fig.17 Plots of variation in peak $(\mu \mathrm{T})$ and variation in field $(\mu \mathrm{T})$ against distance (metres)

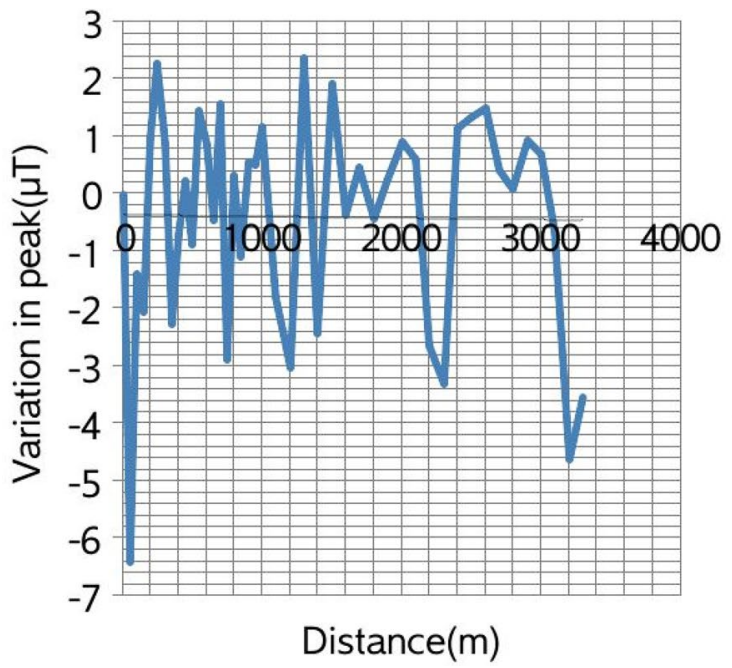

VARIATION IN PEAK $(\mu T)$

Linear (VARIATION IN

$\operatorname{PEAK}(\mu \mathrm{T}))$

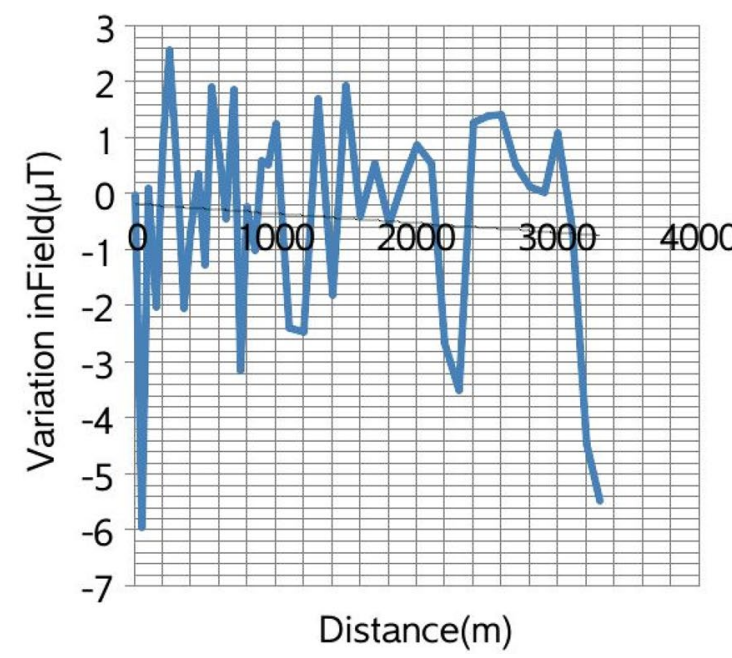

—VARIATION IN FIELD $(\mu \mathrm{T})$

Linear (VARIATION IN FIELD $(\mu T))$

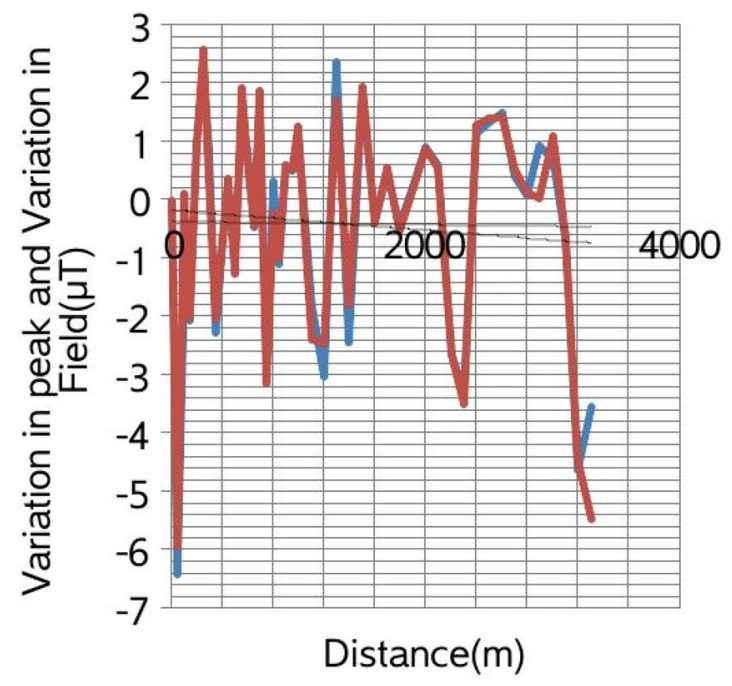

VARIATION IN PEAK $(\mu T)$

VARIATION IN FIELD $(\mu T)$

- Linear (VARIATION IN PEAK $(\mu \mathrm{T}))$

Linear (VARIATION IN FIELD $(\mu T)$ )

Distance(m) 


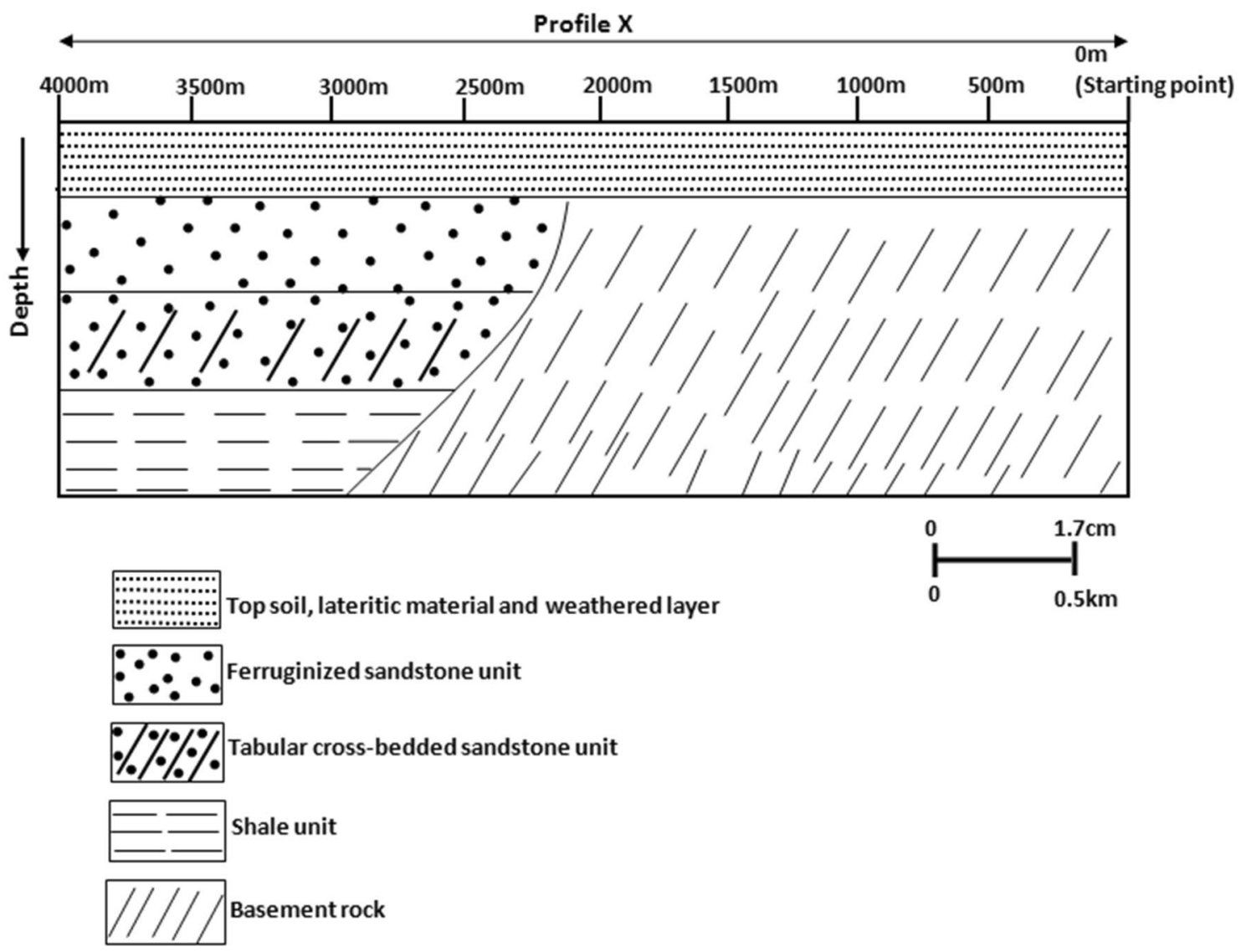

Fig.18 Schematic model of the basement-sedimentary geometry along profile $\mathrm{X}$

established at a much closer interval, particularly for the magnetic survey, in order to obtain a far better subsurface electrical conductivity and magnetic susceptibility information about the area.

It is also suggested that electrical resistivity method should be carried out around the suggested boundary between basement and sedimentary terrains to validate the results of the geophysical surveys utilized in this study.

Funding The authors received no special funding for this research.

\section{Declarations}

Conflict of interest There are no competing interests to declare.

Open Access This article is licensed under a Creative Commons Attribution 4.0 International License, which permits use, sharing, adaptation, distribution and reproduction in any medium or format, as long as you give appropriate credit to the original author(s) and the source, provide a link to the Creative Commons licence, and indicate if changes were made. The images or other third party material in this article are included in the article's Creative Commons licence, unless indicated otherwise in a credit line to the material. If material is not included in the article's Creative Commons licence and your intended use is not permitted by statutory regulation or exceeds the permitted use, you will need to obtain permission directly from the copyright holder. To view a copy of this licence, visit http://creativecommons.org/licenses/by/4.0/.

\section{References}

Adekoya JA, Aluko AF, Opeloye SA (2011) Sedimentological characteristics of Ajali Sandstone in the Benin flank of Anambra Basin. Nigeria Ife J Sci 13(2):327-337

Anifowose AYB, Bamisaye OA, Odeyemi IB (2006) Establishing a solid mineral database for part of Southwestern Nigeria. Geospatial World Newsletter. Available:www.geospatialworld.net

Authors' extract of Auchi and environs (2012)

Egbuniwe IG, Ocan OO (2009) Selection of field work area for teaching/training: Igarra area as an example. In: Lambert-Aikhionbare DO, Olayinka AI (eds) Proceedings of field mapping standardization workshop. University of Ibadan Press, pp 79-95

Geological map of Nigeria, GSN (1994)

Iheanacho PU (2016) Subsurface evaluation of source rock and hydrocarbon potential of the Anambra Basin, South Eastern Nigeria. Dissertation.com Boca Raton, Florida, USA.www.bookpump. com. ISBN 978-1-59942-339-5

Ilegieuno OA, Ighodaro EJ, Sunny RO (2020) Sedimentology and Paleogeography Synthesis of Cretaceous Sediments in Auchi Area of Anambra Basin. J Appl Sci Environ Manage. https://doi.org/10.4314/jasem.v24i5.32 
Kearey P, Brooks M, Hill H (2002) Introduction to geophysical exploration, 3rd edition. Blackwell Science Limited. ISBN: 0-632-04929-4

Nwajide CS (1990) Cretaceous sedimentation and paleogeography of the Central Benue Trough. In: Ofoegbu CO (ed) The Benue Trough structure and evolution. International Monograph Series. Braunschweig, pp 19-38

Obaje NG (2009) Geology and Mineral Resources of Nigeria. Springer, Dordrecht Heidelberg London, p 110. https://doi.org/10.1007/ 978-3-540-92685-6

Odeyemi IB, Rahaman MA (1992) The petrology of a composite syenite dyke in Igarra, Southwestern Nigeria. J Min Geol 28(2):255-263
Olubayo LB (2016) The Main Oil Source Formations of the Anambra Basin, Southeastern Nigeria. Geology Department, University of Ibadan, Nigeria. www.searchanddiscovery.com

Oluseyi AB (2006) Detailed mineralogical study of the Precambrian Basement rocks of Igarra area, Southern Nigeria (Unpublished M.Sc. thesis, Federal University of Technology, Akure, Nigeria)

Tijani MN, Nton ME, Kitagawa R (2010) Textural and geochemical characteristics of the Ajali Sandstone, Anambra basin, SE Nigeria: implication for Its provenance. CR Geosci 342:136-150

Umeji OP, Nwajide CS (2007) Age control and designation of the standard stratotype of Nsukka Formation of Anambra Basin. Southeastern Nigeria J Min Geol 43(2):147-166 